\title{
EFFECT OF PRE-STORAGE HEATING OF BROILER BREEDER EGGS, STORED FOR LONG PERIODS, ON HATCHABILITY AND CHICK QUALITY
}

\author{
H.B. Gharib \\ Department of Animal Production, Faculty of Agriculture, Cairo University, Giza, Egypt \\ Email :( hassangharib2001@gmail.com)
}

\section{SUMMARY}

This study was undertaken to determine if pre-storage heating of broiler breeder eggs, stored for long periods, would improve the hatchability and chick quality. A total of 2400 broiler breeder eggs were obtained from a 46 weeks old commercial flock. Eggs were distributed in a $3 \times 4$ factorial design, with three pre-storage heating durations (0, 6, and 9 hours at $37.5^{\circ} \mathrm{C}$ and $\left.56 \% \mathrm{RH}\right)$ and four storage periods $(4,7,10$ and 14 days at $18^{\circ} \mathrm{C}$ and $\left.75 \% \mathrm{RH}\right)$. This adds up to twelve treatments with 20 replicates, of 10 eggs each. After storage, eggs were simultaneously incubated under the usual incubation conditions. Eggs were weighed before heating, after storage and before transfer to the hatcher, to determine the weight loss. Fertility and hatchability of both fertile and total eggs were determined. Embryo mortality was determined in non-hatched eggs. All hatched chicks were weighed and examined to score them for quality. The results indicated that long egg storage period resulted in a significant increase in egg weight loss during storage and after 18 days of incubation, and total egg weight loss. The longer the pre-storage heating duration was significantly increased the egg weight loss percentage during storage. Hatchability and chick quality results of eggs stored from 7 tol 4 days were significantly less than eggs stored for 4 days. The 6 hours pre-storage heating treatment, significantly increased the hatchability and chick quality of eggs as compared to the non-heated or 9 hours heated eggs. There were significant pre-storage heating $\times$ egg storage length interactions for egg weight loss, hatchability of total and fertile eggs, embryonic mortality and chick quality. When eggs were stored for more than seven days, pre-storage heating eggs for six hours significantly improved the hatchability and chick quality results, as compared to non-heated or heated for 9 hours eggs. In conclusion, pre-storage heating of commercial broiler breeder eggs for six hours at $37.5^{\circ} \mathrm{C}$ and $56 \%$ RH could be used by the poultry industry as a method to improve hatchability and chick quality of eggs stored for longer periods than 7 days.

Keywords: Pre-storage heating, storage periods, embryonic mortality, hatchability, chick quality

\section{INTRODUCTION}

Storage of hatching eggs is a common practice in commercial breeder farms and hatcheries; the total length of egg storage varies between a few days and several weeks. However, the storage duration depends on the supply of hatching eggs, maximum hatchery capacity, and the variable market demand for 1-day old chicks in the poultry industry. Normally, commercial hatcheries set their eggs after 3 to 5 days of storage, but in some situations, a hatchery needs to increase the storage period beyond 7 days. Egg storage period beyond 7 days is associated with a lag in embryo development (Christensen et al., 2001), altered metabolic rate (Christensen et al., 2001 and Fasenko et al., 2009), a delay in hatch time (Ruiz and Lunam, 2002 and Tona et al., 2003), impairing embryo development and livability (Christensen et al., 2002, Elibol et al., 2002 and Van de Ven, 2004), and decline in hatchability (Tona et al., 2004, Petek and Dikmen, 2006 and Yassin et al., 2008). In addition, Tona et al. (2003\& 2004) and Willemsen et al. (2008) determined the negative effects of prolonged egg storage on chick quality in terms of physical parameters on the day of hatch such as appearance, activity, and quality of the navel area. Besides body weight and other physical parameters, used by Tona et al. (2003), it is also possible to determine the negative effect of long egg storage period on chick quality at hatch with a quantitative method, such as chick length (Wolanski et al., 2004 and Molenaar et al., 2008).

The negative effects of long storage of hatching eggs result from the deterioration of internal egg quality. With long storage time, albumen $\mathrm{pH}$ increases whereas, albumen height decreases (Lapao et al., 1999) and yolk sac membrane elasticity decreases (Jones and Musgrove, 2005). Embryonic mortality during storage is highly related to egg weight loss, and increases with storage time (Nahm, 2001 and Reijrink et al., 2010). Reijrink et al. (2008) stated that the negative effects of prolonged egg storage may be caused by changes in the embryo or in the egg characteristics, or by both.

During storage, as eggs remain at cold temperature, below physiological zero $\left(20-21^{\circ} \mathrm{C}\right)$, embryos stop developing at a stage characterize by the complete formation of zona pellucid. However, later, as temperature increase during incubation, embryo development is resumed (Fasenko et al., 2001a). Higher embryo livability and hatchability, and shorter incubation period were observed when the hypoblast stage is achieved before long storage periods (Fasenko et al., 2001a and Reijrink et al., 
2009). Before storage, heating chicken eggs for six hours (Fasenko et al., 2001a), turkey eggs for 12 hours (Fasenko et al., 2001b) and quail eggs for 8 hours (Petek and Dikmen, 2004) allows the complete formation of hypoblast. Therefore, pre-storage heating could improve the incubation results of eggs stored for long periods (Fasenko et al., 2001a \& b, Renema et al., 2006 and Silva et al., 2008).

When working with long storage periods, the adoption of management practices such as storing the egg with the thin tip down, egg turning during storage and pre-storage heating should be considered to reduce the negative effects on the incubation yield (Rocha et al., 2013). It was reported that pre-heating of poultry eggs, before storage, resulted in more live chicks and a lower level of embryonic mortality compared to eggs that were not heated (Lourens, 2002, Petek and Dikmen, 2004 \& 2006 and Marandure et al., 2012). On the other hand, Reijrink et al. (2009) stated that pre-storage heating can have a negative as well as a positive effect on hatchability and chick quality. They suggested that pre-storage heating affects embryo viability and therefore survival rate of the embryo during early incubation. Moreover, Reijrink et al. (2010) reported that prestorage heating of eggs (for seven hours) was probably not necessary to make the embryos more resistant to prolonged egg storage (15 days) and did, therefore, not improve hatchability or chick quality.

According to Fasenko et al. (2001b), the effect of pre-storage heating on hatchability results, when storage time is prolonged, depends on the developmental stage of the embryo after pre-storage heating. Various pre-incubation storage practices, of hatching eggs, have different considerable influences on the hatching results. However, the effect of prestorage heating of hatching eggs on chick quality is not studied extensively. Therefore, the specific goal of this study was to determine if pre-storage heating would improve the hatchability and chick quality of broiler breeder eggs stored for long periods.

\section{MATERIAL AND METHODS}

\section{Experimental design}

A total of 2520 , clean, not cracked or missshaped, hatching eggs produced by a 46-week-old Hubbard Classic broiler breeder flock were collected from a commercial farm and were transported to the experimental hatchery in four groups (630 eggs each). Out of the total number of eggs, 2400 eggs were distributed in a $3 \times 4$ factorial design, with three pre-storage heating durations $(0,6$, and 9 hours at $37.5^{\circ} \mathrm{C}$ and $\left.56 \% \mathrm{RH}\right)$ and four storage periods (4, 7, 10 and 14 days at $18^{\circ} \mathrm{C}$ and $75 \% \mathrm{RH}$ ), summing up twelve treatments with 20 replicates of 10 eggs each. Eggs were hand collected directly from the nests during the second daily collection in order to ensure lay time uniformity. Eggs were collected between five and seven hours after light was switched on, when the outside temperature was around $20^{\circ} \mathrm{C}$. In order to incubate all eggs at the same time, eggs were collected in four groups, one group every four days, representing the four storage periods $(4,7,10$ and 14 days). Therefore, the first group corresponded to eggs stored for 14 days; the second to eggs stored for ten days; the third to eggs stored for seven days and the fourth to eggs stored for four days. Out of the 630 eggs obtained in each collection, 30 were chosen at random (Table 1) to evaluate internal and external egg quality, using egg weight, shell thickness, and percentages of albumen, yolk and shell weights.

\section{General Management}

Immediately after collection, the eggs were disinfected by simple fumigation with $7.7 \mathrm{~g}$ paraformaldehyde/m3 area for 15 minutes (Khan et $a l ., 2012$ ), and then transported to the experimental hatchery. The eggs were then disinfected by formaldehyde fumigation for $20 \mathrm{~min}$, identified, weighed in pools of ten eggs, and randomly distributed into the treatments. Average time between egg collection and beginning of the experiment was four hours. Pre-storage heating for six or nine hours (depending on treatment) was carried out in a Chick Master ${ }^{\circledR}$ incubator at an average temperature of $37.5^{\circ} \mathrm{C}$ and relative humidity of $56 \%$. The heating timing started when the temperature reached $37.5^{\circ} \mathrm{C}$. After heating, eggs were kept at room temperature for one hour. Eggs were then stored in a storage room at $18^{\circ} \mathrm{C}$ and $75 \%$ relative humidity for the periods corresponding to the treatments. After storage, eggs were maintained at $25^{\circ} \mathrm{C}$ and $75 \%$ relative humidity for six hours before incubation. Eggs were incubated in a Chick Master $₫$ incubator for 442 hours (18 days and 10 hours) at $37.5^{\circ} \mathrm{C}$, average temperature, and $56.5 \%$ relative humidity. Eggs were turned hourly. At 442 hours of incubation, eggs were transferred to a Chick Master $₫$ hatcher at $36.6^{\circ} \mathrm{C}$, average temperature, and $61.2 \%$ average relative humidity.

\section{Measured parameters}

All eggs were weighed, in pools of ten eggs, before; heating, incubation, and transfer to the hatcher to evaluate the egg weight loss. Egg weight loss was determined during the storage periods, after 18 days of incubation and the total of two periods. After 18 days of incubation, all eggs were candled to calculate the fertility percentage, for each replicate (10 eggs each). Eggs with evidence of living embryos were transferred from the incubation trays to the hatcher trays. After the hatch was completed, un-hatched eggs were removed, broken and submitted to embryo diagnosis to determine the percentages of early, intermediate, and late embryo mortality ( 1 to 7,8 to 14 and 15 to 21 days of embryo development, resp.). Early dead embryos were differentiated by the absence of an egg tooth; the intermediate dead were differentiated by the presence of an egg tooth, the beginning of feathers 
and the yolk sac outward the body cavity. The late dead were differentiated by evidence of the yolk sac entering the body cavity and the beak positioned to pip the air cell. Piped (piped and could not hatch) and rotten (contaminated eggs and non-specific embryonic mortality) chicks were also determined. Total embryonic mortality was determined as the amount of the all dead embryos.

Hatchability of fertile eggs and total hatchability percentages were determined at 21 days of incubation, for each replicate, as follow:

Hatchability of fertile eggs $\%=$ (number of hatched chicks/ fertile eggs set)*100

Total hatchability \% = (number of hatched chicks/ total eggs set)*100

All hatched chicks were weighed and examined to score them for quality (Tona et al., 2003; 2004). Chicks of good quality (saleable chicks, grade A) were defined as being clean, dry and free from deformities (no skin lesion, well-formed beak, normal conformation of legs), completely sealed navel, and no yolk sac or residual membrane protruding from the navel area (Tona et al., 2004). The un-saleable chicks were examined for classification into grade $\mathrm{B}$ or $\mathrm{C}$ according to the previous traits (Grade B = Bad Chicks; Grade $\mathrm{C}=$ Very bad Chicks.). All day-old chicks were scored with a Tona score as described by Tona et al. (2003). Chick length was determined according to Willemsen et al. (2008).

\section{Statistical analyses}

Data were submitted to two way analysis of variance using the General Linear Model procedure of SAS version 8 (1999). The main factors were storage period and pre storage heating duration. Percentage data were subjected to arc sine transformation prior to analysis. Mean values were compared using Duncan's Multiple Range Test (Duncan, 1955) when significant differences existed. Significance was set at $\mathrm{P}<0.05$.

\section{RESULTS}

Egg characteristics, on a subsample of eggs ( $\mathrm{n}=$ 30 per group) designated to be stored for $4-14$ days, were conducted to determine if egg component variations existed between the eggs collected maximum 10 days apart. Fresh egg weight, albumen weight $(\%)$, yolk weight $(\%)$, dried shell weight $(\%)$, and eggshell thickness were not significantly influenced by the egg collection period (Table 1).

\section{Egg weight and Egg weight loss}

The main effects of egg storage period and prestorage heating duration on fresh egg weight and egg weight loss percentage are presented in Table (2). No significant differences were found in fresh egg weights among the different storage period or pre-storage heating duration treatments. However, significant effect of storage period was observed on egg weight loss percentages during storage period, after 18 days of incubation, and during the two periods together (total egg weight loss \%). The longer the storage period or the pre-storage heating duration, the higher the egg weight loss percentage was observed (Table 2). Eggs that were heated for 9 hours, pre-storage, had significantly higher egg weight loss percentage, during the first 18 days of incubation and total egg weight loss, as compared to those non-heated or heated for six hours.

The results indicated that there were significant interactions between storage period and pre-storage heating duration on egg weight loss percentages during storage and after 18 days of incubation, and total egg weight loss percentage (Table 2). Egg weight loss percentage during storage increased as a function of storage period at any preheating duration. However, eggs heated for six or nine hours and stored for ten days had higher weight loss percentage, during storage, as compared to nonheated eggs. During first $18 \mathrm{~d}$ of incubation, egg weight loss percentage was not different among preheating duration within storage period, except when eggs were stored for 14 days. Where, eggs heated for 6 or 9 hours had significantly higher egg weight loss compared to the non-heated eggs. However, within pre-heating treatments, longer storage period resulted in significantly higher percentage of egg weight loss during incubation. Results of total egg weight loss percentage indicated that eggs stored for 4,7 or 10 days were not influenced by heating duration. Whereas, within the 14 days storage group, eggs heated for nine hours had higher weight loss percentage as compared to non-heated eggs. On the other hand, within preheating treatments, total egg weight loss percentage was proportional to storage period.

\section{Fertility, hatchability and embryonic mortality}

The results of the apparent fertility, hatchability of total and fertile eggs, and embryonic mortality are shown in Table (3). There were no significant effects of the egg storage period, pre-storage heating duration or the interaction between them on the apparent fertility percentage.

Hatchability of fertile and total eggs was significantly affected by both the experimental factors and the interaction between them. Longer period of egg storage resulted in a linear significant decrease in the hatchability of fertile and total eggs, independent of the pre-heating duration. In general, eggs heated for six hours, pre-storage, had significantly higher hatchability of fertile and total eggs, as compared to non-heated eggs or those heated for nine hours. Pre-storage heating of eggs for six hours significantly increased hatchability in eggs stored for more than 4 days (Table 3 ). Within the four days storage period, eggs heated for nine hours had significantly lower hatchability as compared to those non-heated. Eggs heated for six hours, pre- 
storage, had higher hatchability when stored for four or seven days as compared to eggs stored for 10 or 14 days (Table 3 ).

Early and late embryonic mortality (1-7 and 1521 days of incubation, respectively), piped eggs, and total embryonic mortality percentages were significantly influenced by the experimental treatments (Table 3). Rotten eggs percentage was significantly affected only, by the storage period. On the other hand, intermediate embryonic mortality (814 days of incubation) was not influenced by the storage periods or pre-storage heating duration. Higher percentages of early, late and total embryonic mortality; and piped and rotten eggs were associated with longer egg storage period. When eggs were sorted for 14 days, they had significantly the highest early and total embryonic mortality rates, and piped and rotten eggs percentages (Table 3). This was followed by the shorter periods of storage groups.

Significantly higher late embryonic mortality rate was observed in eggs stored for 10 and 14 days, as compared to the other storage period groups. Regardless of storage period, pre-heating eggs for six hours resulted in significantly lower percentages of early, late and total embryonic mortality when compared to the non-heated eggs or heated for nine hours (Table 3 ).

Significant interactions were also detected between the pre-storage heating duration and storage period on all embryonic mortality rates, and piped and rotten eggs percentages (Table 3 ). The results indicated that pre-storage heating eggs for 6 hours significantly decreased early embryonic mortality, when eggs were stored for seven and 14 days. Within all storage periods, pre-storage heating eggs for nine hours led to higher intermediate embryonic mortality rate, as compared to those not heated or heated for six hours.

Pre-storage heating eggs for nine hours resulted in significantly higher rates of late embryonic mortalities when eggs were stored for four or ten days, and significantly lower late embryonic mortality in eggs stored for 14 days, as compared to those not heated. Pre-storage heating eggs for six hours and storing them for 14 days resulted in significant decrease in the percentages of piped and rotten eggs, as compared to the non-heated eggs (Table 3). When eggs were stored for more than four days, total embryonic mortality rates were significantly lower when eggs were pre-storage heated for six hours, as compared to those not heated or heated for nine hours.

\section{Chick Quality}

Different indicators for measuring the chick quality were used. These indicators included commercial chick quality grade, Tona score, chick body weight, and chick length at 1 day old. All chick quality traits studied were significantly affected by both storage period and pre-storage heating duration (Table 4). Long egg storage period was associated with the decrease in grade A chicks percentage, Tona score chick quality, body weight, and chick length, at one day old. Whereas, Grade B and C chicks percentages were significantly higher for eggs stored for more than four days, as compared to those stored for only four days.

Pre-storage heating of eggs for six hours resulted in significant improvement in all chick quality traits, as compared to non-heated eggs. This improvement was not significant when compared to pre-storage heated eggs for nine hours (Table 4), except grade C chicks, which were significantly higher in nine hours group.

There were significant interactions between the storage period and pre-storage incubation duration for chicks' grade. The obtained data indicated that the chicks produced from heated eggs for six hours and stored for 4-14 days had significantly higher percentages of grade A chicks, as compared to nonheated eggs (Table 4). The significant improvement in grade A chicks percentage in the six hours heating group, as compared to nine hours heating group was observed, only, when eggs were stored for four or ten days (Table 4).

When storage period was 10 or 14 days, the chicks produced from pre-storage heated eggs for 6 hours had significantly higher Tona scores than those hatched from non-heated eggs or heated for nine hours. When eggs were stored for 10 or 14 days, heating eggs for six or nine hours produced chicks with significantly heavier weight, as compared to non-heated ones. The chicks hatched from six hours heated eggs and stored for 7 to 14 days were significantly longer, as compared to those hatched from non-heated or nine hours heated eggs.

Table 1. Internal and external egg quality profiles of the four groups of eggs before starting the treatments

\begin{tabular}{cccccc}
\hline $\begin{array}{c}\text { Egg collection } \\
\text { period }\end{array}$ & Egg weight $(\mathbf{g})$ & Albumen (\%) & Yolk (\%) & Shell (\%) & $\begin{array}{c}\text { Eggshell } \\
\text { thickness (mm) }\end{array}$ \\
\hline First & $66.08 \pm 0.95$ & $60.95 \pm 0.68$ & $29.94 \pm 1.12$ & $9.11 \pm 0.35$ & $0.43 \pm 0.033$ \\
Second & $66.29 \pm 0.85$ & $61.10 \pm 0.57$ & $29.79 \pm 1.05$ & $9.11 \pm 0.31$ & $0.43 \pm 0.034$ \\
Third & $66.38 \pm 0.77$ & $61.38 \pm 0.61$ & $29.60 \pm 1.09$ & $9.02 \pm 0.28$ & $0.43 \pm 0.032$ \\
Fourth & $66.57 \pm 0.89$ & $61.43 \pm 0.65$ & $29.52 \pm 1.11$ & $9.05 \pm 0.38$ & $0.43 \pm 0.035$ \\
$\boldsymbol{P}$ & 0.572 & 0.298 & 0.630 & 0.121 & 0.550 \\
\hline
\end{tabular}

-No significant differences, within trait, among the egg collection groups were observed 
Table 2.The effects of egg storage period, pre-storage heating duration and their interaction on fresh egg weight and egg weight loss percentage ${ }^{1}$ of broiler breeder eggs

\begin{tabular}{|c|c|c|c|c|}
\hline \multirow{2}{*}{$\begin{array}{c}\text { Main treatment } \\
\text { effects }\end{array}$} & \multicolumn{4}{|c|}{ Trait } \\
\hline & $\begin{array}{l}\text { Fresh egg } \\
\text { weight (g) }\end{array}$ & $\begin{array}{l}\text { Egg weight } \\
\text { loss during } \\
\text { storage }(\%) \\
\end{array}$ & $\begin{array}{l}\text { Egg weight loss } \\
\text { after } 18 \text { days of } \\
\text { incubation }(\%)\end{array}$ & $\begin{array}{c}\text { Total egg } \\
\text { weight loss } \\
(\%) \\
\end{array}$ \\
\hline \multicolumn{5}{|c|}{ Egg storage period (days) } \\
\hline 4 & 66.4 & $0.75^{\mathrm{d}^{*}}$ & $12.84^{\mathrm{d}}$ & $13.59^{\mathrm{d}}$ \\
\hline 7 & 66.5 & $0.95^{\mathrm{c}}$ & $13.03^{\mathrm{c}}$ & $13.99^{\mathrm{c}}$ \\
\hline 10 & 66.6 & $1.21^{\mathrm{b}}$ & $13.31^{\mathrm{b}}$ & $14.52^{\mathrm{b}}$ \\
\hline 14 & 66.7 & $1.51^{\mathrm{a}}$ & $13.69^{\mathrm{a}}$ & $15.20^{\mathrm{a}}$ \\
\hline SEM $^{* *}$ & 0.086 & 0.020 & 0.021 & 0.028 \\
\hline $\boldsymbol{P}$ & 0.786 & 0.026 & 0.032 & 0.028 \\
\hline \multicolumn{5}{|l|}{ Pre-storage heating } \\
\hline $\mathbf{0}$ & 66.5 & $1.01^{\mathrm{b}}$ & $13.18^{\mathrm{b}}$ & $14.19^{\mathrm{b}}$ \\
\hline 6 & 66.4 & $1.13^{\mathrm{a}}$ & $13.09^{\mathrm{b}}$ & $14.22^{\mathrm{b}}$ \\
\hline 9 & 66.5 & $1.17^{\mathrm{a}}$ & $13.21^{\mathrm{a}}$ & $14.38^{\mathrm{a}}$ \\
\hline SEM & 0.093 & 0.021 & 0.032 & 0.035 \\
\hline $\boldsymbol{P}$ & 0.802 & 0.010 & 0.019 & 0.023 \\
\hline \multicolumn{5}{|c|}{ Storage period $x$ pre-storage } \\
\hline \multicolumn{5}{|c|}{ heating duration interaction } \\
\hline 4days * 0 hours & 66.5 & $0.67^{\mathrm{d}}$ & $12.90^{\mathrm{e}}$ & $13.57^{\mathrm{e}}$ \\
\hline 4 days $* 6$ hours & 66.5 & $0.77^{\mathrm{d}}$ & $12.82^{\mathrm{e}}$ & $13.59^{\mathrm{e}}$ \\
\hline 4 days $* 9$ hours & 66.4 & $0.82^{\mathrm{d}}$ & $12.80^{\mathrm{e}}$ & $13.62^{\mathrm{e}}$ \\
\hline 7 days $* 0$ hours & 66.4 & $0.91^{\mathrm{cd}}$ & $13.08^{\mathrm{d}}$ & $13.99^{\mathrm{d}}$ \\
\hline 7 days $* 6$ hours & 66.3 & $0.96^{\mathrm{cd}}$ & $13.04^{\mathrm{d}}$ & $14.00^{\mathrm{d}}$ \\
\hline 7 days $* 9$ hours & 66.5 & $0.99^{\mathrm{cd}}$ & $13.00^{\mathrm{d}}$ & $13.99^{\mathrm{d}}$ \\
\hline 10 days $* 0$ hours & 66.6 & $1.02^{\mathrm{c}}$ & $13.35^{\mathrm{c}}$ & $14.37^{\mathrm{c}}$ \\
\hline 10 days * 6 hours & 66.5 & $1.31^{\mathrm{b}}$ & $13.28^{\mathrm{c}}$ & $14.59^{\mathrm{c}}$ \\
\hline 10 days $* 9$ hours & 66.6 & $1.30^{\mathrm{b}}$ & $13.30^{\mathrm{c}}$ & $14.60^{\mathrm{c}}$ \\
\hline 14 days $* 0$ hours & 66.4 & $1.47^{\mathrm{ab}}$ & $13.63^{b}$ & $15.10^{\mathrm{b}}$ \\
\hline 14 days $* 6$ hours & 66.5 & $1.49^{\mathrm{ab}}$ & $13.72^{\mathrm{a}}$ & $15.21^{\mathrm{ab}}$ \\
\hline 14 days $* 9$ hours & 66.4 & $1.57^{\mathrm{a}}$ & $13.73^{\mathrm{a}}$ & $15.30^{\mathrm{a}}$ \\
\hline SEM & 0.071 & 0.199 & 0.032 & 0.039 \\
\hline $\boldsymbol{P}$ & 0.651 & 0.001 & 0.001 & 0.002 \\
\hline
\end{tabular}

\footnotetext{
Means, within columns, for the main treatment effects or the interaction effects, with no common superscript, differ significantly $(\mathrm{P} \leq 0.05)$.

${ }^{* *} \mathrm{SEM}=$ Stander error of the mean.

${ }^{1}$ Egg weight loss percentage estimated as a percentage of fresh egg weight.
} 
Table 3. The effects of egg storage period, pre-storage heating duration and their interaction on apparent fertility, hatchability and embryonic mortality of broiler breeder eggs

\begin{tabular}{|c|c|c|c|c|c|c|c|c|c|}
\hline \multirow{3}{*}{ Main treatment effects } & \multicolumn{9}{|c|}{ Trait } \\
\hline & \multirow[b]{2}{*}{$\begin{array}{l}\text { Apparent } \\
\text { fertility } \%\end{array}$} & \multirow{2}{*}{$\begin{array}{c}\text { Hatchability } \\
\text { of fertile eggs } \\
\%\end{array}$} & \multirow{2}{*}{$\begin{array}{c}\text { Hatchability } \\
\text { of total eggs } \\
\%\end{array}$} & \multicolumn{6}{|c|}{ Embryonic mortality \% } \\
\hline & & & & Early & Mid $^{1}$ & late & Piped egg & Rotten egg & Total \\
\hline \multicolumn{7}{|l|}{ Egg storage period (days) } & $0.6^{\mathrm{b}}$ & $0.8^{\mathrm{b}}$ & $7.2^{\mathrm{d}}$ \\
\hline $\begin{array}{l}4 \\
7\end{array}$ & 90.0 & $89.6^{\mathrm{b}}$ & $80.1^{\mathrm{b}}$ & $4.4^{\mathrm{b}}$ & 0.6 & $3.6^{\mathrm{b}}$ & $1.1^{\mathrm{b}}$ & $0.6^{\mathrm{b}}$ & $10.3^{\mathrm{c}}$ \\
\hline $\begin{array}{c}7 \\
10\end{array}$ & 90.2 & $87.3^{c}$ & $77.3^{\mathrm{c}}$ & $4.4^{\mathrm{b}}$ & 0.7 & $5.8^{\mathrm{a}}$ & $1.0^{\mathrm{b}}$ & $0.7^{\mathrm{b}}$ & $12.6^{\mathrm{b}}$ \\
\hline $\begin{array}{l}10 \\
14\end{array}$ & 90.3 & $82.4^{\mathrm{d}}$ & $73.4^{\mathrm{d}}$ & $7.6^{\mathrm{a}}$ & 1.1 & $5.0^{\mathrm{a}}$ & $2.3^{\mathrm{a}}$ & $1.8^{\mathrm{a}}$ & $17.8^{\mathrm{a}}$ \\
\hline SEM $^{\text {14* }}$ & 0.08 & 0.31 & 0.30 & 0.26 & 0.25 & 0.29 & 0.27 & 0.21 & 0.712 \\
\hline$P$ & 0.205 & 0.001 & 0.001 & 0.001 & 0.351 & 0.002 & 0.006 & 0.020 & 0.001 \\
\hline Pre-storage heating duration (hours at $37.5^{\circ} \mathrm{c}$ ) & 90.3 & $86.8^{\mathrm{b}}$ & $77.5^{\mathrm{b}}$ & $5.9^{\mathrm{a}}$ & 0.6 & $4.4^{\mathrm{a}}$ & $1.6^{\mathrm{a}}$ & 0.8 & $13.3^{\mathrm{a}}$ \\
\hline 0 & 90.0 & $91.2^{\mathrm{a}}$ & $81.2^{\mathrm{a}}$ & $3.4^{\mathrm{b}}$ & 0.6 & $3.2^{\mathrm{b}}$ & $1.0^{\mathrm{b}}$ & 0.7 & $8.9^{\mathrm{b}}$ \\
\hline $\mathbf{6}$ & 90.2 & $86.2^{\mathrm{b}}$ & $77.1^{\mathrm{b}}$ & $5.2^{\mathrm{a}}$ & 1.3 & $4.8^{\mathrm{a}}$ & $1.1^{\mathrm{b}}$ & 1.4 & $13.8^{\mathrm{a}}$ \\
\hline $\begin{array}{c}9 \\
\text { SEM }\end{array}$ & 0.091 & 0.421 & 0.410 & 0.34 & 0.32 & 0.29 & 0.21 & 0.30 & 0.61 \\
\hline $\begin{array}{c}\text { SEM } \\
P\end{array}$ & 0.264 & 0.031 & 0.030 & 0.023 & 0.456 & 0.018 & 0.022 & 0.608 & 0.017 \\
\hline \multicolumn{10}{|l|}{ Storage period $\mathrm{x}$ pre-storage heating duration } \\
\hline interaction & 91.3 & $93.8^{\mathrm{a}}$ & $84.9^{\mathrm{a}}$ & $2.6^{\mathrm{e}}$ & $0.3^{\mathrm{d}}$ & $2.0^{\mathrm{g}}$ & $1.2^{\mathrm{bc}}$ & $0.1^{\mathrm{d}}$ & $6.2^{\mathrm{d}}$ \\
\hline 4days * 0 hours & 89.4 & $94.2^{\mathrm{a}}$ & $83.7^{\mathrm{ab}}$ & $2.7^{\mathrm{e}}$ & $0.4^{\mathrm{d}}$ & $1.7^{\mathrm{g}}$ & $0.2^{\mathrm{e}}$ & $0.8^{\mathrm{c}}$ & $5.8^{\mathrm{d}}$ \\
\hline 4 days $* 6$ hours & 90.7 & $90.3^{\mathrm{b}}$ & $81.5^{\mathrm{b}}$ & $3.4^{\mathrm{d}}$ & $1.6^{\mathrm{a}}$ & $2.7^{\mathrm{f}}$ & $0.5^{\mathrm{d}}$ & $1.5^{\mathrm{bc}}$ & $9.7^{\mathrm{c}}$ \\
\hline 4 days $* 9$ hours & 89.7 & $87.8^{\mathrm{cd}}$ & $78.0^{\mathrm{cd}}$ & $6.2^{\mathrm{c}}$ & $0.3^{\mathrm{d}}$ & $3.8^{\mathrm{e}}$ & $1.5^{\mathrm{b}}$ & $0.4^{\mathrm{cd}}$ & $12.2^{\mathrm{bc}}$ \\
\hline 7 days $* 0$ hours & 90.0 & $93.6^{\mathrm{a}}$ & $83.7^{\mathrm{ab}}$ & $2.3^{\mathrm{f}}$ & $0.3^{\mathrm{d}}$ & $2.4^{\mathrm{fg}}$ & $0.9^{\mathrm{bc}}$ & $0.5^{\mathrm{cd}}$ & $6.4^{\mathrm{d}}$ \\
\hline 7 days $* 6$ hours & 90.3 & $87.8^{\mathrm{cd}}$ & $78.7^{\mathrm{c}}$ & $4.8^{\mathrm{d}}$ & $1.3^{\mathrm{a}}$ & $4.5^{\mathrm{de}}$ & $0.8^{\mathrm{c}}$ & $0.8^{\mathrm{c}}$ & $12.2^{\mathrm{bc}}$ \\
\hline 7 days * 9 hours & 89.7 & $86.4^{\mathrm{d}}$ & $76.5^{\mathrm{d}}$ & $5.5^{\mathrm{cd}}$ & $0.6^{\mathrm{c}}$ & $5.5^{\mathrm{c}}$ & $1.2^{\mathrm{b}}$ & $0.8^{\mathrm{c}}$ & $13.6^{\mathrm{b}}$ \\
\hline 10 days $* 0$ hours & 90.6 & $89.1^{\mathrm{c}}$ & $78.9^{c}$ & $3.1^{\mathrm{de}}$ & $0.6^{\mathrm{c}}$ & $5.2^{\mathrm{c}}$ & $1.2^{\mathrm{bc}}$ & $0.8^{\mathrm{c}}$ & $10.9^{c}$ \\
\hline 10 days * 6 hours & 90.3 & $86.4^{\mathrm{d}}$ & $76.6^{\mathrm{d}}$ & $4.5^{\mathrm{de}}$ & $0.9^{\mathrm{b}}$ & $7.2^{\mathrm{a}}$ & $0.5^{\mathrm{d}}$ & $0.5^{\mathrm{cd}}$ & $13.6^{\mathrm{b}}$ \\
\hline 10 days $* 9$ hours & 90.6 & $79.1^{\mathrm{e}}$ & $70.6^{\mathrm{e}}$ & $9.3^{\mathrm{a}}$ & $1.0^{\mathrm{b}}$ & $6.2^{\mathrm{b}}$ & $2.6^{\mathrm{a}}$ & $1.8^{\mathrm{b}}$ & $20.9^{\mathrm{a}}$ \\
\hline 14 days $* 0$ hours & 90.0 & $87.8^{\mathrm{cd}}$ & $78.4^{\mathrm{cd}}$ & $5.6^{\mathrm{cd}}$ & $0.9^{\mathrm{b}}$ & $3.4^{\mathrm{de}}$ & $1.5^{\mathrm{b}}$ & $0.8^{\mathrm{c}}$ & $12.2^{\mathrm{bc}}$ \\
\hline 14 days $* 6$ hours & 90.4 & $80.3^{\mathrm{e}}$ & $71.2^{\mathrm{e}}$ & $8.0^{\mathrm{b}}$ & $1.3^{\mathrm{a}}$ & $4.8^{\mathrm{d}}$ & $2.7^{\mathrm{a}}$ & $2.9^{\mathrm{a}}$ & $19.7^{\mathrm{a}}$ \\
\hline 14 days $* 9$ hours & 0.72 & 1.03 & 0.99 & 0.46 & 0.11 & 0.34 & 0.23 & 0.32 & 1.06 \\
\hline SEM & 0.783 & 0.001 & 0.001 & 0.001 & 0.031 & 0.001 & 0.009 & 0.018 & 0.009 \\
\hline $\boldsymbol{P}$ & & & & & & & & & \\
\hline
\end{tabular}


Table 4. The effects of egg storage period, pre-storage heating duration and their interaction on chick quality of broiler breeder eggs

\begin{tabular}{|c|c|c|c|c|c|c|}
\hline \multirow{3}{*}{$\begin{array}{l}\text { Main treatment } \\
\text { effects }\end{array}$} & \multicolumn{6}{|c|}{ Trait } \\
\hline & \multicolumn{3}{|c|}{ Chick quality $(\text { Commercially })^{1}$} & \multirow{2}{*}{$\begin{array}{c}\text { Tona } \\
\text { score } \\
\text { chick } \\
\text { quality }^{5}\end{array}$} & \multicolumn{2}{|c|}{$\begin{array}{c}\text { Day old chick quality } \\
\text { measurements }\end{array}$} \\
\hline & $\begin{array}{c}\text { Grade } \\
\mathbf{A}^{2}\end{array}$ & $\begin{array}{c}\text { Grade } \\
\mathbf{B}^{\mathbf{3}}\end{array}$ & $\begin{array}{c}\text { Grade } \\
\text { C }^{4}\end{array}$ & & $\begin{array}{c}\text { Body } \\
\text { Weight } \\
(\mathrm{g}) \\
\end{array}$ & $\begin{array}{c}\text { Chick } \\
\text { length } \\
\text { (cm) }\end{array}$ \\
\hline \multicolumn{7}{|c|}{ Egg storage period (days) } \\
\hline $\mathbf{4}$ & $96.6^{\mathrm{a}^{*}}$ & $2.8^{\mathrm{d}}$ & $0.6^{\mathrm{c}}$ & $95.4^{\mathrm{a}}$ & $46.0^{\mathrm{a}}$ & $19.3^{\mathrm{a}}$ \\
\hline 7 & $92.8^{\mathrm{b}}$ & $5.0^{\mathrm{c}}$ & $2.2^{\mathrm{b}}$ & $91.8^{\mathrm{b}}$ & $45.2^{\mathrm{b}}$ & $19.2^{\mathrm{b}}$ \\
\hline 10 & $91.4^{\mathrm{b}}$ & $6.7^{\mathrm{b}}$ & $1.9^{\mathrm{b}}$ & $85.4^{\mathrm{c}}$ & $43.3^{c}$ & $19.2^{\mathrm{b}}$ \\
\hline 14 & $87.8^{\mathrm{c}}$ & $8.6^{\mathrm{a}}$ & $3.6^{\mathrm{a}}$ & $82.0^{\mathrm{d}}$ & $41.8^{\mathrm{d}}$ & $19.2^{\mathrm{b}}$ \\
\hline SEM $^{* *}$ & 0.71 & 0.24 & 0.29 & 1.95 & 0.09 & 0.01 \\
\hline $\boldsymbol{P}$ & 0.009 & 0.012 & 0.021 & 0.001 & 0.019 & 0.021 \\
\hline \multicolumn{7}{|c|}{$\begin{array}{l}\text { Pre-storage heating duration } \\
\quad\left(\text { hours at } 37.5^{\circ} \mathrm{c}\right)\end{array}$} \\
\hline $\mathbf{0}$ & $90.7^{\mathrm{b}}$ & $7.0^{\mathrm{a}}$ & $2.3^{\mathrm{a}}$ & $86.0^{\mathrm{b}}$ & $43.8^{b}$ & $19.2^{\mathrm{b}}$ \\
\hline 6 & $93.6^{\mathrm{a}}$ & $4.7^{\mathrm{b}}$ & $1.7^{\mathrm{b}}$ & $91.4^{\mathrm{a}}$ & $44.3^{\mathrm{a}}$ & $19.3^{\mathrm{a}}$ \\
\hline 9 & $92.1^{\mathrm{ab}}$ & $5.6^{\mathrm{b}}$ & $2.3^{\mathrm{a}}$ & $88.5^{\mathrm{ab}}$ & $44.2^{\mathrm{a}}$ & $19.2^{\mathrm{b}}$ \\
\hline SEM & 0.63 & 0.55 & 0.30 & 1.36 & 0.05 & 0.01 \\
\hline $\boldsymbol{P}$ & 0.015 & 0.032 & 0.023 & 0.032 & 0.019 & 0.016 \\
\hline \multicolumn{7}{|c|}{ Storage period $x$ pre-storage } \\
\hline \multicolumn{7}{|c|}{ heating duration interaction } \\
\hline 4days $* 0$ hours & $96.0^{\mathrm{b}}$ & $3.1^{\mathrm{g}}$ & $0.9^{\mathrm{de}}$ & $94.3^{\mathrm{ab}}$ & $46.0^{\mathrm{a}}$ & $19.3^{\mathrm{a}}$ \\
\hline 4 days $* 6$ hours & $98.0^{\mathrm{a}}$ & $1.5^{\mathrm{h}}$ & $0.5^{\mathrm{e}}$ & $96.5^{\mathrm{a}}$ & $46.0^{\mathrm{a}}$ & $19.3^{\mathrm{a}}$ \\
\hline 4 days $* 9$ hours & $95.8^{\mathrm{b}}$ & $3.7^{\mathrm{fg}}$ & $0.5^{\mathrm{e}}$ & $95.3^{\mathrm{ab}}$ & $45.9^{\mathrm{a}}$ & $19.2^{\mathrm{b}}$ \\
\hline 7 days $* 0$ hours & $91.7^{\mathrm{e}}$ & $6.1^{\mathrm{d}}$ & $2.2^{\mathrm{c}}$ & $91.5^{\mathrm{c}}$ & $45.2^{\mathrm{b}}$ & $19.2^{\mathrm{b}}$ \\
\hline 7 days * 6 hours & $93.8^{\mathrm{cd}}$ & $4.1^{\mathrm{f}}$ & $2.1^{\mathrm{c}}$ & $93.1^{\mathrm{bc}}$ & $45.3^{\mathrm{b}}$ & $19.3^{\mathrm{a}}$ \\
\hline 7 days $* 9$ hours & $93.0^{\mathrm{d}}$ & $4.7^{\mathrm{ef}}$ & $2.3^{\mathrm{bc}}$ & $90.8^{\mathrm{c}}$ & $45.2^{\mathrm{b}}$ & $19.2^{\mathrm{b}}$ \\
\hline 10 days $* 0$ hours & $89.8^{\mathrm{f}}$ & $8.1^{\mathrm{b}}$ & $2.1^{\mathrm{c}}$ & $83.5^{\mathrm{e}}$ & $42.7^{\mathrm{d}}$ & $19.2^{\mathrm{b}}$ \\
\hline 10 days $* 6$ hours & $93.6^{\mathrm{cd}}$ & $5.1^{\mathrm{e}}$ & $1.4^{\mathrm{d}}$ & $88.3^{\mathrm{cd}}$ & $43.7^{\mathrm{c}}$ & $19.3^{\mathrm{a}}$ \\
\hline 10 days $* 9$ hours & $91.1^{\mathrm{ef}}$ & $6.8^{\mathrm{cd}}$ & $2.1^{\mathrm{c}}$ & $84.4^{\mathrm{e}}$ & $43.6^{c}$ & $19.2^{\mathrm{b}}$ \\
\hline 14 days $* 0$ hours & $85.4^{\mathrm{g}}$ & $10.6^{\mathrm{a}}$ & $4.0^{\mathrm{a}}$ & $74.6^{\mathrm{f}}$ & $41.2^{\mathrm{f}}$ & $19.2^{\mathrm{b}}$ \\
\hline 14 days * 6 hours & $89.1^{\mathrm{f}}$ & $8.2^{\mathrm{b}}$ & $2.7^{\mathrm{b}}$ & $87.8^{\mathrm{cd}}$ & $42.1^{\mathrm{e}}$ & $19.3^{\mathrm{a}}$ \\
\hline 14 days $* 9$ hours & $88.8^{\mathrm{f}}$ & $7.1^{\mathrm{c}}$ & $4.1^{\mathrm{a}}$ & $83.6^{\mathrm{e}}$ & $42.0^{\mathrm{e}}$ & $19.2^{\mathrm{b}}$ \\
\hline SEM & 0.52 & 0.24 & 0.21 & 0.39 & 0.11 & 0.01 \\
\hline $\boldsymbol{P}$ & 0.013 & 0.019 & 0.025 & 0.019 & 0.001 & 0.014 \\
\hline
\end{tabular}

\footnotetext{
Means, within columns, for the main treatment effects or the interaction effects, with no common superscript, differ significantly $(\mathrm{P} \leq 0.05)$.

SEM $=$ Stander error of the mean

2- Grade A = Good commercial Chicks.

1- According to commercial hatchery standards.

4- Grade $\mathrm{C}=$ Very bad Chicks.

3- Grade B = Bad Chicks.

5- Tona Score according to Tona et al. (2003).
} 


\section{DISCUSSION}

In the present study, egg external and internal quality traits were not significantly influenced by collection day (maximum 10 days apart) indicating that egg collection day did not directly or indirectly affect the evaluated parameters. Similar results were observed by Silva et al. (2008). They reported that internal and external egg qualities of eggs were not different among three different collection days; eggs were collected in three five-day intervals throughout 14 days.

The present results indicated that egg weight losses, during storage, were significantly affected by the main effects of the pre-storage heating duration and length of egg storage. Egg weight loss was proportional to the storage period. Egg weight loss percentage in the 14-day storage group was double that of the four-day storage group. Differences in egg weight losses, prior to incubation, were significantly higher with pre-storage heating eggs for nine hours, followed by six hours heating, as compared to the non-heated eggs. These results were expected because an exposure to long-time storage and heat treatment would increase the opportunity for water evaporation from the eggs. This finding is in agreement with that of Silva et al. (2008) and Reijrink et al. (2010).

In the present study, the long storage period or pre-storage heating eggs did not affect apparent fertility. Fertility should not have been affected by the two main treatments because fertilization would or would not have occurred before the eggs were exposed to the treatments. Similar suggestions were reported by Fasenko et al. (2001a) in chicken eggs, and Petek and Dikmen (2004) in quail eggs. They found that the differences for the apparent fertility among the main groups of pre-storage heating and storage duration were not significant.

The current results revealed that egg storage for more than four or seven days markedly impaired incubation results due to higher egg weight loss, as shown by the lower hatchability; higher total embryonic mortality percentage, as well as lower chick quality. These results are in agreement with previous reports on broiler breeder's eggs (Fasenko et al., 2001a \& b, Fasenko, 2007 and Silva et al., 2008). They observed lower hatchability and higher embryonic mortality percentage of embryos stored for 14 days as compared to 4 days of storage. These results may be due to that some embryos, from eggs stored for a long period, and could not start developing immediately after normal incubation temperatures were provided or they develop at a slower rate (Fasenko et al., 2001a). Haque et al. (1996) observed lower embryo metabolic rate, particularly during the last stage of embryo development, as well as changes in the circulatory system during embryogenesis as storage period increased. This could explain the increase in late embryo mortality and poorer chick quality found in eggs stored for more than seven days, in the present study. Long periods of storage may also increase the probability of bacterial contamination, as cuticle quality deteriorates (De Reu et al., 2006). Our results confirmed this suggestion, with higher rotten eggs percentage and worse chick quality associated with eggs stored for 14 days.

The deleterious effects of long-term egg storage on chick quality could be due to the reduction of embryo weight. This is an indication of decreased embryo quality that could affect hatch quality (Hamidu et al., 2011). Previously, embryos from broiler eggs, stored for 14 days, displayed a reduced growth rate, lower hatchability and poor chick quality compared with eggs stored for 4 days (Fasenko et al., 2001a). In other studies, embryos from eggs stored for long periods had a slower metabolic rate than those from eggs stored for shorter periods (Fasenko and Robinson, 2001), slower growth of heart and liver (Christensen et al., 2001) and a decline in relative lung weight (Yalçin and Siege, 2003) which resulted in poor chick quality.

Strategies are needed, that allows for the storage of eggs for considerably longer periods with minimum deterioration in hatchability and chick quality, thus increasing productivity of commercial hatcheries. In the present experiment, eggs were, prestorage, heated $\left(0,6\right.$, or 9 hours at $37.5^{\circ} \mathrm{C}$ and $56 \%$ $\mathrm{RH})$, and were subsequently stored at $18^{\circ} \mathrm{C}$ and $75 \%$ $\mathrm{RH}$, thereby ensuring that the embryos developmental hypoblast stage was achieved by heating and was maintained throughout the storage period. Fasenko et al. (1992) stated that embryo stage is not changed by storage period when storage temperature is below embryo development physiological zero $\left(20-21^{\circ} \mathrm{C}\right)$.

Eggs stored for 7 - 14 days and were heated for six hours prior to storage presented significantly lower total embryonic mortality and higher hatchability of fertile eggs, as compared to those not heated or heated for nine hours. Heating eggs for six hours before storing for 10 or 14 days significantly improved the chick quality, as compared to not heated or heated eggs for nine hours. There was a significant depressive effect of heating eggs for nine hours on the hatchability of total and fertile eggs in eggs stored for four days, whereas this effect was not significant when the eggs were stored longer. The results related to pre-storage heating in long period stored eggs were in agreement with previous reports (Fasenko et al., 2001a \& b, Petek and Dikmen, 2004 and Silva et al., 2008).

The improvement in the incubation yield and chick quality in pre-storage heating eggs for six hours, as compared to those not heated or heated for nine hours may be related to the embryos stage and total number of viable embryonic cells, prior to storage. Reijrink et al. (2010) reported that, prestorage heating eggs for seven hours increased the 
stage of embryonic development, the total number of embryonic cells, and the total number of viable embryonic cells. How far an embryo develops depends on warming duration and temperature. Reijrink et al. (2009) showed that the ability of an embryo to survive prolonged egg storage may depend on the cell activity at a particular stage of development but may also depend on the number of viable embryonic cells. When the number of viable embryonic cells is low, at the onset of incubation, due to cell death during storage, particular steps in the embryo development may be impeded. This may result in abnormal development or embryonic death. Therefore, pre-storage heating of eggs for 6 hours may be considered as a good practice to improve incubation results of eggs stored for short, intermediate, and long periods.

The storage immediately after egg collection increased early embryonic mortality and reduced hatchability, probably due to the higher number of embryos in a pre- gastrula stage, which would be more sensitive to cool temperature and storage stress than the embryos at gastrula stage (Fiuza et al., 2006). In a study of Fasenko et al. (2001a), after their pre-storage heating treatment of 6 hours, $76.7 \%$ of the embryos were at developmental stage EG13 (hypoblast stage). Hypoblast formation is the initial stage of gastrulation, ensuring their survival during prolonged storage. They hypothesized that embryos at developmental stage EG12 or EG13 are less sensitive to prolonged egg storage than embryos that are less or further advanced. At EG13 stage, the embryo has completed hypoblast formation, and cell migration and differentiation is minimal (Bellairs, 1986).

Conversely, pre-storage heating of eggs for nine hours caused worse hatchability in eggs stored for 4 14 days, as compared to six hours, probably because it took the embryos to a developmental stage after EG13 (Fasenko et al., 2001a and Malecki et al., 2005). In embryos further advanced, damage caused by prolonged storage times might be irreversible and might cause embryonic mortality. Fasenko et al. (2001b) hypothesized that embryos advanced to the developmental stage EG12 or EG13 are more resistant for prolonged egg storage than embryos further advanced. The advanced stage of gastrulation is characterized by active migration and differentiation, and the embryo is highly sensitive to change in temperature over long periods. In addition, eggs heated for nine hours before 14 days of storage had high egg weight loss after storage, which may be related to their low hatchability. These findings indicated that pre-storage heating eggs treatment for nine hours was not a proper for eggs stored for $4-14$ days.

Pre-storage heating of eggs for 6 hours improved chick quality in terms of grad A chicks percentage, chick weight and length and Tona score, independent of storage time, as compared to the non-heated eggs.
These results are in agreement with those reported by Yalçin and Siegel (2003) and Reijrink et al. (2009). Reijrink et al. (2010) reported that Pre-storage heat treatment improved chick quality on the day of hatch in terms of chick length. They stated that, it is possible that this difference in chick length between the control and pre-storage heated groups is caused by a difference in hatch time. Pre-storage heating increased embryonic development and therefore chicks of the pre-storage heated group hatched earlier than chicks of the control group (Yalçin and Siegel, 2003 and Reijrink et al., 2009). This might explain why chick length and weight of the pre-storage heated eggs was higher at the moment of measurement than that of the non-heated eggs (Willemsen et al., 2008).

\section{CONCLUSIONS}

In conclusion, pre-storage heating of commercial broiler breeder eggs, for six hours, could be used by the industry as a method to improve hatchability of hatching eggs stored for seven days or more. Further research is needed to precisely determine the number of hours of pre-storage heating required to obtain maximum hatchability and chick quality. Meanwhile, it should be kept in mind that the economic cost of pre-storage heating must be evaluated in comparison with its beneficial effects. Data from the present experiment suggested that a six hours pre-storage heating is optimum. In addition, it should be noted that the present study examined specific storage periods of 4 - 14 days; exposure of eggs to other egg storage periods, season and frequency of egg collection could alter the effectiveness of the prestorage heating treatments.

\section{REFERENCES}

Bellairs, R., 1986. The primitive streak, Anatomy and Embryology (Berl.), 174:1-14.

Christensen, V. L., M. J. Wineland, G. M. Fasenko, and W. E. Donaldson, 2001. Egg storage effects on plasma glucose and supply and demand tissue glycogen concentrations of broiler embryos. Poultry Science, 80:1729-1735.

Christensen, V., M. Wineland, G. Fasenko, W. E. Donaldson, 2002. Egg storage alters weight of supply and demand organs of broiler chicken embryos. Poultry Science, 81:1738- 1743.

De Reu, K., K. Grijspeerdt, Messens, W. Heyndrickx, M. Uyttendaele, J. Debevere and L. Herman, 2006. Eggshell factors influencing eggshell penetration and whole egg contamination by different bacteria, including Salmonella enteritidis. International Journal of Food Microbiology, 112:253-260.

Duncan, D. B., 1955. Multiple ranges and multiple F tests. Biometrics, 11: 1-42. 
Elibol, O., S. D. Peak, and J. Brake, 2002. Effect of flock age, length of egg storage, and frequency of turning during storage on hatchability of broiler hatching eggs. Poultry Science, 81: 945-950.

Fasenko, G. M., 2007. Egg storage and the embryo. Poultry Science, 86:1020-1024.

Fasenko, G.M., F.E. Robinson and R.T. Hardin, 1992. Variability in preincubation embryonic development in domestic fowl. 2. Effects of duration of egg storage period. Poultry Science, 71:2129-2132.

Fasenko, G.M. and F. E. Robinson, 2001. What happens to the growth and metabolism of broiler embryos and chicks when you store hatching eggs for long periods prior to incubation? Alberta Poultry Research center newsletter, 10 (2), University of Alberta, Edmunton, Canada.

Fasenko, G. M., F. E. Robinson, A. I. Whelan, K. M. Kremeniuk, and J. A. Walker, 2001a. Prestorage incubation of long-term stored broiler breeder eggs: 1. Effects on hatchability. Poultry Science, 80:1406-1411.

Fasenko, G. M., V. L. Christensen, M. J. Wineland, and J. N. Pettite, 2001b. Examining the effects of prestorage incubation of turkey breeder eggs on embryonic development and hatchability of eggs stored for four or fourteen days. Poultry Science, 80:132-138.

Fasenko, G. M., F. E. Robinson, and V. L. Christensen, 2009. Effects of long term storage on the egg, embryo and chick. Avian Biological Research, 2:73-79.

Fiuza, M., L. Lara, C. Aguilar, B. Ribeiro and N. Baiao, 2006. Efeitos das condicoes ambientais no period entre a postura e o armazenmento de ovosde matrizes pesadas sobre o rendimento de incubacao. Arquivo Brasileiro de Medicina Veterinaria e Zootecnia, 58: 408-413.

Hamidu, J., Z. Uddin , M. Li, G. Fasenko, L.Guan , and D. Barreda, 2011. Broiler egg storage induces cell death and influences embryo quality. Poultry Science, 90:1749-1757.

Haque, M. A, J.T. Pearson, C.L. Hou and H. Tazawa, 1996. Effects of preincubation egg storage on embryonic functions and growth. Respiration Physiology, 103:89-98.

Jones, D.R. and M.T. Musgrove, 2005. Effects of extended storage on egg quality factors. Poultry Science, 84:1774-1777.

Khan, M. J, A. Abbas, M. Ayaz, M. Naeem, S. Qadir, M. S. Akhter and W. Zia, 2012. Effect of pre-heating and turning during storage period on hatchability and post hatch performance of broilers. Journal of Biological Sciences, 1: 1-6

Lapao, C., L. T. Gama, and M. Chaveiro Soares, 1999. Effects of broiler breeder age and length of egg storage on albumen characteristics and hatchability. Poultry Science, 78:640-645.
Lourens, S., 2002. Heating of hatching eggs prestorage improves hatchability. World Poultry Science, 18:24-25.

Malecki, I.A., J.O. Horbanczuk, C. E. Reed and G.B. Martin, 2005. The ostrich (Struthio camelus) blastoderm and embryo development following storage of eggs at various temperatures. British Poultry Science, 46: 652-660.

Marandure, T., G. Matondi, G. B. Nyamushamba and B. Ganyani, 2012. Effect of duration of preheating broiler breeder eggs on hatchability, egg weight and chick uniformity post hatch. Research Journal of Agriculture and Environmental Management, 1: 001-005. www.apexjournal.org/RJAEM.

Molenaar, R., I. A. M. Reijrink, R. Meijerhof, and H. van den Brand, 2008. Relationship between hatchling length and weight on later productive performance in broilers. World's Poultry Science Journal, 64:599-603.

Nahm, K.H., 2001. Effects of storage length and weight loss during incubation on the hatchability of ostrich eggs (Struthio camuelus). Poultry Science, 80:1667-1670.

Petek, M. and S. Dikmen, 2004. The effect of prestorge incubation of quail breeder eggs on hatchability and subsequent growth performance of progeny. Animal Research, 53:527-534.

Petek, M. and S. Dikmen, 2006. The effect of prestorage incubation and length of storage of broiler breeder eggs on hatchability and subsequent growth performance of progeny. Czech Journal of Animal Science, 51:73-77.

Reijrink, I. A. , D. Berghmans , R. Meijerhof , B. Kemp and $H$. van den Brand, 2010. Influence of egg storage time and preincubation warming profile on embryonic development, hatchability, and chick quality. Poultry Science, 89:12251238.

Reijrink, I. A. M., R. Meijerhof, B. Kemp, and H. van den Brand., 2008. The chicken embryo and its micro environment during egg storage and early incubation. World's Poultry Science Journal, 64:581- 598.

Reijrink, I. A. M., R. Meijerhof, B. Kemp, E. A. M. Graat, and H. van den Brand, 2009. Influence of pre-storage incubation on embryonic development, hatchability, and chick quality. Poultry Science, 88:2649-2660.

Renema, R. A., J. J. R. Feddes, K. L. Schmid, M. A. Ford, and A. R. Kolk, 2006. Internal egg temperature in response to pre-incubation warming in broiler breeder and turkey eggs. Journal of Applied Poultry Research, 15:1-8.

Rocha, J., N. Baiao, V. Brabosa, M. Pompeu, M. Fernandes, L. Lara, C. Matias and J. Batista, 2013. Negative effects of fertile egg storage on the egg and the embryo and suggested hatchery management to minimize such problems. World's Poultry Science Journal, 69: 35- 44. 
Ruiz J. and C.A. Lunam, 2002. Effect of preincubation storage conditions on hatchability, chick weight at hatch and hatching time in broiler breeders. British Poultry Science, 43:374-383.

SAS Institute, 1999. SAS user's guide: statistics, version 8. (Cary, NC, SAS Institute).

Silva, F.H.A.; D.E Faria; K.A.A. Torres; D.E. Faria Filho; A.D.D. Coelho and V.J.M. Savino. 2008. Influence of egg pre-storage heating period and storage length on incubation results. Brazilian Journal of Poultry Science, 10:17-22.

Tona, K., F. Bamelis, B. De Ketelaere, V. Bruggeman, V. M. B. Moreas, J. Buyse, O. Onagbesan, and E. Decuypere, 2003. Effects of egg storage time on spread of hatch, chick quality, and chick juvenile growth. Poultry Science, 82:736-741.

Tona, K., O. Onagbesan, B. De Ketelaere, E. Decuypere, and V. Bruggeman, 2004. Effects of age of broiler breeders and egg storage on egg quality, hatchability, chick quality, chick weight, and post-hatch growth to forty-two days. Journal of Applied Poultry Research, 13:10-18.
Van de Ven, L., 2004. Storage of hatching eggs in the production process. International Hatchery Practices, 18, 27-31.

Willemsen, H., N. Everaert, A. Witters, L. De Smit, M. Debonne, F. Verschuere, P. Garain, D. Berckmans, E. Decuypere, and V. Bruggeman, 2008. Critical assessment of chick quality measurements as an indicator of post hatch performance. Poultry Science, 87:2358-2366.

Wolanski, N. J., E. J. Luiten, R. Meijerhof, and A. L. J. Vereijken, 2004. Yolk utilization and chick length as parameters for embryo development. Avian Poultry Biology Review, 15:233-234.

Yalçin, S., and P. B. Siegel, 2003. Developmental stability of broiler embryos in relation to length of egg storage prior to incubation. Journal of Poultry Science, 40: 298-308.

Yassin, H., A. G. J. Velthuis, M. Boerjan, J. Van Riel, and R. B. M. Huirne, 2008. Field study on broiler eggs hatchability. Poultry Science, 87:2408-2417.

تأثير التدفئة قبل التخزين لبيض دجاج امهات انتاج اللحم المخزن لفترات طويلة على نسبة الفقس و جودة الكتاكيت الناتجة
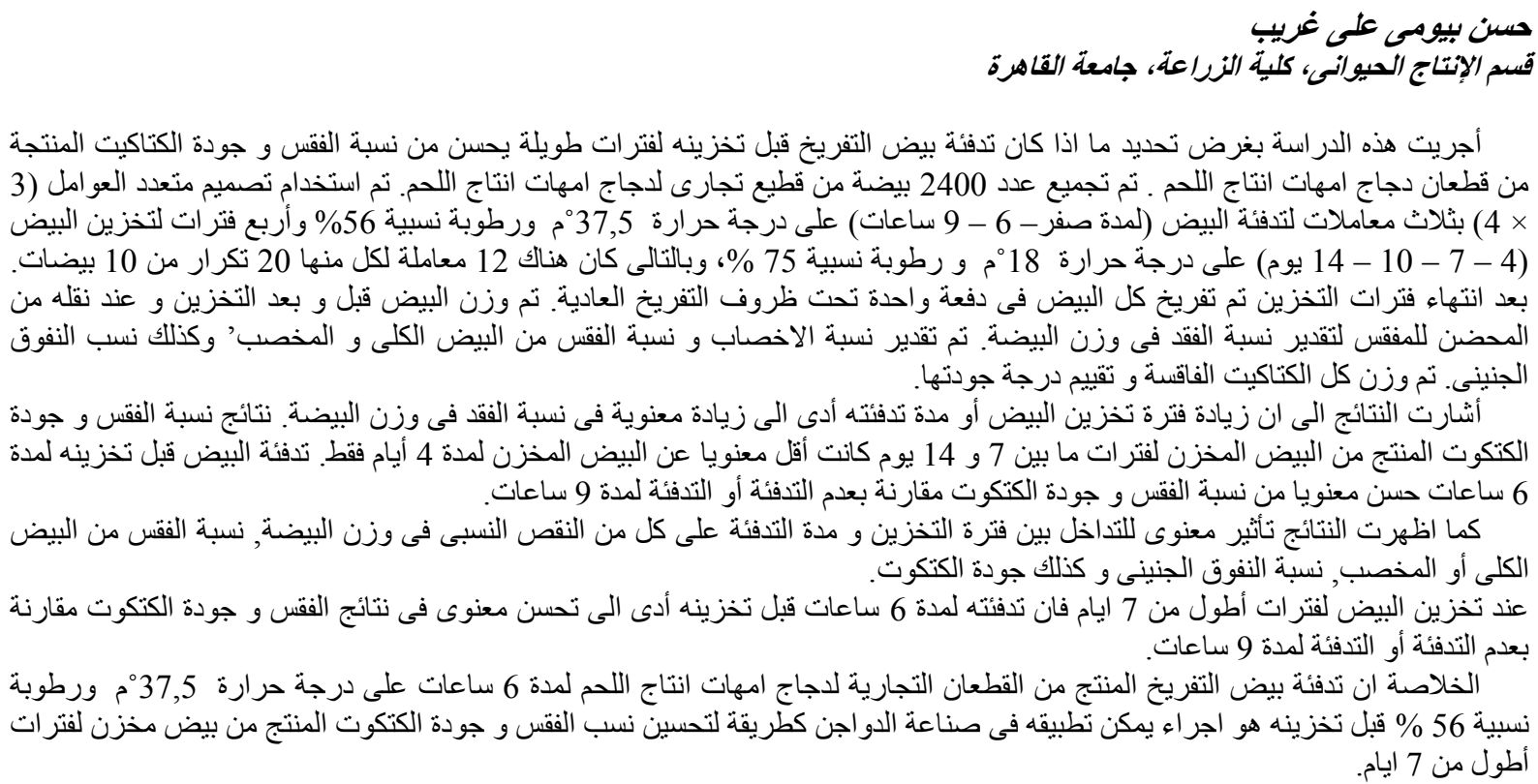